\title{
How do I Imagine Orthodontic World in 2035?
}

\author{
Dr Swati Saraswata Acharya,' Dr Pritam Mohanty,.2 Dr Pavithra Rao³ \\ Reader, Dept of Orthodontics, 'Institute of Dental Sciences, ${ }^{2 K a l i n g a ~ I n s t i t u t e ~ o f ~ D e n t a l ~ S c i e n c e s, ~}{ }^{3 H}$ itech Dental College, \\ Bhubaneswar, India
}

Correspondence: Dr Swati Acharya; Email: swati.acharya.tmdc@gmail.com

\section{ABSTRACT}

The future orthodontic world is so bright that we definitely 'need to wear shades'. We are infiltrating a new era of orthodontics accoutered with modern technologies. However, ever growing operating costs, greater patient expectations and indictments against orthodontists are few concerns which need to be addressed. Increased information technology skills, three-dimensional image superimposition methods, custom designed treatments, tooth movement control systems, digital models and evidencebased orthodontics will be the new outlook in the next twenty years. Orthodontic research is entering an epoch of exhilaration. Trends, issues and new evolutions can come together in startling ways. Scenarios are the recitals about the future that combine these forces of change in various ways. Genome wide coalition studies are vital to further the evidence base to practice orthodontics in the coming years.

Key words: evidence-based, future, genome, orthodontic research, technologies

Renowned science fiction author Isaac Asimov once asserted that "whoever writes science fiction cannot help making predictions - not of what will happen but of what may happen". Since researchers are often required to scan the present in order to shed light on the future, we could modestly compare ourselves to science fiction writers. This is the outlook I intend to adopt in this article.

Orthodontics now is very different from ten years ago since it is now a lot denser to establish the flow of patients. Before 2008, orthodontists had an easier time to assemble their patients when money was more free-flowing, and the more patients that you acquire then more referrals you get down the line. I tried this low-fee orthodontic practice in my working class neighborhood, and I can authenticate that several other orthodontic practitioners have tried this approach without much triumphs including myself. I lowered the fees and then treated the patients well, yet they did not refer others to me. Presently, I am doing better in my "top $20 \%$ neighborhood" because these people actually see the value and dedication in my work and hospitality, and are not just looking for the low treatment fees, and definitely they do refer others to me because they have had a good experience. Various other problems that newer orthodontists encounter are that the general practitioner referrals tend to come from your own age. For those new graduate general practitioners tend to do clear aligners or hire in-house orthodontists since there are many ortho graduates passing out every year. Orthodontic claims are increasing according to Dental Protection and $20 \%$ of these claims in 2010 were related to aligner systems.

Today there are lots of arising challenges wrecking the sanguine scenario of orthodontists around the globe. They circle from the lack of opportunities for the newly qualified specialists to insecurity where the issue of systembased orthodontics is highly questionable around the globe. In recent years for different reasons, the world of future orthodontics appear less blooming.' How reasonable is it for an Orthodontist in the market today? In what geographic area are you located? What do you realize is the greatest challenge to a freshly passed out Orthodontist today? What specialty would you propose for a graduating dental student? These are few concerns which make me ponder the dull side of orthodontics in near future. The other rosy side would be advancing Information Technology skills which will prove significant in daily practice.

Digital models are now a tangible reality at good prices. Digital study models not only to speed up preparation along with analysis but also the expense of preserving plaster models will be unreasonably high around the world. Three-dimensional printers will be applicable whenever physical models are required. Three-dimensional image 
superimposition methods will be used more rapidly. Orthodontic postgraduates will have access to much better designed studies and more of evidence-based practice.' Technological advances will make convenient custom-designed treatment protocols and therefore we will be able to view more patients in less time with a high class of excellence. Orthodontic practice will undergo various changes as well. Information Technology will boost patient care by assisting the work flow. Tooth movement control systems will sharpen orthodontists in taking the necessary therapeutic measures. Patients will interact more in making even more informed decisions about what the treatment plan and objective has in store for them. Furthermore, professionals are incorporating them into clinical practice, and thus increasing the quality and standard of their presentations for patients. Focusing on the patient's perception of what matters, is of concern and vital during treatment will increase both patient and parent satisfaction towards the results. Armed with modern technologies like $\mathrm{CBCT}$ radiography, advanced diagnostic and treatment concepts, modern self-ligating lingual appliances with TADs and soft tissue lasers, orthodontists will be better able to respond to patient demands and needs. All issues mentioned here will lead to a proper quality of orthodontic services which will rise for the benefit of orthodontists. Many of us may be imagining the relationship between this prevarication and today's orthodontics. The solution is that they are intensely entwined. Obviously, I only mentioned one amongst many other possible future scenarios. However, my vision is already materializing and the fact that many young orthodontic professionals who are not aware of it should think it as a reason of concern. Thus, as said by Asimov,' the predictions I made may not be what will happen, but what is likely to happen'. Whatever the future brings, preparing for it necessitates proper training and good education.

Ever-increasing operating costs, increased patient expectations, and actions against orthodontists are some of the common and major concerns. The 'golden age' of orthodontics has crossed. Hence, there is a reason to be optimistic about our future orthodontic world and even remain excited about what contemporary practice has to give our patients in improving their oral health issues. We should be prepared to understand the factors that attribute to patient satisfaction and dissatisfaction. And, definitely patient satisfaction with orthodontic treatment solely depends on the 'quality of treatment result'.

\section{OJN}

\section{REFERENCE}

1. Keim RG. What is the future of orthodontics? Am J Orthod Dentofac Orthop 1991; 99:22 